\title{
Neutrino Nucleus Reactions and Nucleosynthesis in Stars
}

\author{
Toshio Suzuki* \\ Department of Physics, Nihon University \\ Center for Nuclear Study, University of Tokyo \\ E-mail: suzuki@phys.chs.nihon-u.ac.jp
}

\section{Takashi Yoshida}

National Astronomical Observatory of Japan

\section{Satoshi Chiba}

Advanced Science Research Center, Japan Atomic Energy Agency

\section{Michio Honma}

Center for Mathematical Sciences, University of Aizu

\section{Koji Higashiyama}

Department of Physics, Chiba Institute of Technonolgy

\section{Hideyuki Umeda}

Department of Astronomy, School of Science, University of Tokyo

\section{Ken'ichi Nomoto}

Department of Astronomy, Graduate School of Science, and Institute for the Physics and Mathematics of the Universe, University of Tokyo

\section{Toshitaka Kajino}

National Astronomical Observatory of Japan

Department of Astronomy, Graduate School of Science, University of Tokyo

\section{Takaharu Otsuka}

Department of Physics and Center for Nuclear Study, University of Tokyo RIKEN 
Neutrino-nucleus reactions in light nuclei as well as in $\mathrm{Ni}$ and $\mathrm{Fe}$ isotopes are investigated based on new shell model Hamiltonians for $p$-shell and $f p$-shell. Spin and magnetic properties of nuclei have been considerably improved by the Hamiltonians. Neutrino-induced reaction cross sections in ${ }^{12} \mathrm{C},{ }^{4} \mathrm{He}, \mathrm{Ni}$ and $\mathrm{Fe}$ isotopes are obtained with the use of the new Hamiltonians, and they are found to be enhanced compared with those by conventional Hamiltonians as well as previous calculations. The production yields of ${ }^{7} \mathrm{Li}$ and ${ }^{11} \mathrm{~B}$ during supernova explosions are found to be enhanced. Production of other light elements ${ }^{6} \mathrm{Li},{ }^{9} \mathrm{Be},{ }^{10} \mathrm{Be}$ and ${ }^{10} \mathrm{~B}$ is also discussed. Neutral current and charge-exchange reactions on $\mathrm{Ni}$ and $\mathrm{Fe}$ isotopes are studied for supernova neutrinos. Neutrino processes during supernova explosions, especially reactions on ${ }^{56} \mathrm{Ni}$, are pointed out to make important roles in the production of heavy elements such as Mn in population III stars. Implications of enhanced cross sections on the production yields of the heavy elements are discussed.

10th Symposium on Nuclei in the Cosmos

July 27 - August 1, 2008

Mackinac Island, Michigan, USA

${ }^{*}$ Speaker.

(C) Copyright owned by the author(s) under the terms of the Creative Commons Attribution-NonCommercial-ShareAlike Licence. 


\section{Neutrino-Induced Reactions on ${ }^{12} \mathrm{C}$ and ${ }^{4} \mathrm{He}$ and Synthesis of Light Elements}

Recently, new shell model Hamiltonians for $p$-shell nuclei were obtained[1, 2] based on the Cohen-Kurath[3] and Millener-Kurath[4] interactions. A new Hamiltonian, SFO[2], properly takes into account important roles of spin-isospin interactions, especially, the tensor interaction. Systematic improvements in the descriptions of the magnetic moments of the $p$-shell nuclei as well as Gamow-Teller (GT) transitions, for example, in ${ }^{12} \mathrm{C}$ and ${ }^{14} \mathrm{~N}$ have been obtained[2]. These improvements in the Hamiltonian are essential for the study of neutrino-nucleus reactions, which are induced mainly by GT and spin-dipole transitions.

We study neutrino-induced reactions on ${ }^{12} \mathrm{C}$ based on the new improved shell model Hamiltonian, SFO[2]. Experimental cross section data are available for ${ }^{12} \mathrm{C}$. The exclusive charge-exchange and neutral current reaction cross sections for ${ }^{12} \mathrm{C}$ induced by the GT transition are well reproduced by the SFO Hamiltonian for the DAR neutrinos[5]. The inclusive charge-exchange reaction is also well reproduced with a quenching for the axial-vector coupling constant, $g_{A}^{\text {eff }} / g_{A}^{\text {free }}=0.70(0.90)$ for the $2^{-}\left(0^{-}, 1^{-}\right.$etc.) states, which is consistent with the electron scattering data [6].
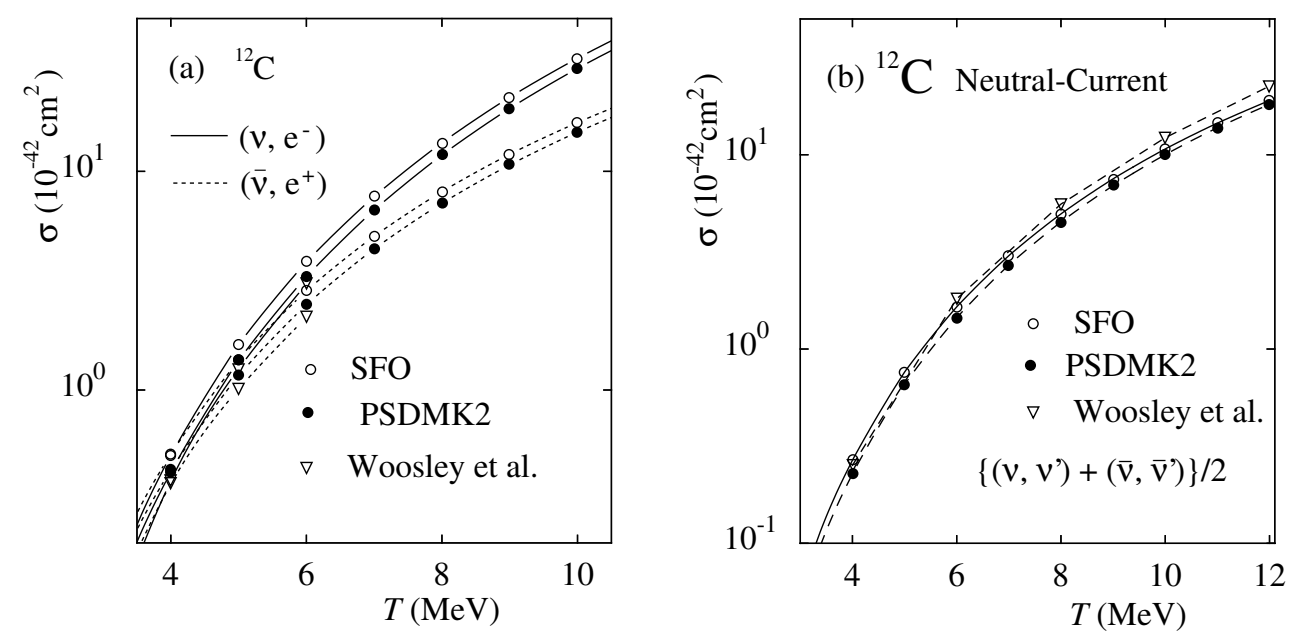

Figure 1: Calculated (a) charge-exchange and (b) neutral current reaction cross sections for ${ }^{12} \mathrm{C}$ induced by supernova neutrinos with temperature $T$ obtained by shell model calculations with the use of the SFO and PSDMK2 Hamiltonians. Previous results in ref. [7] are also shown for comparison.

Reactions induced by supernova neutrinos have been investigated, and the cross sections are found to be enhanced compared with those by the conventional shell model Hamiltonians (e. g. PSDMK2[4]) as well as previous calculations[7] as shown in Fig. 1. Reaction cross sections for the $\gamma$ decay, proton, neutron, deuteron, ${ }^{3} \mathrm{He}, \mathrm{t}$ and $\alpha$ knock-out processes including multi-particle knock-out processes are obtained for supernova neutrinos with temperature $T$. The branching ratios for the processes have been calculated by the Hauser-Feshbach theory.

Now, we study neutrino-induced reaction on ${ }^{4} \mathrm{He}$ as they play important roles in the nucleosynthesis of light elements during supernova explosions. Calculated cross sections for neutrinoinduced reactions on ${ }^{4} \mathrm{He}$ are shown in Fig. 2 for the WBP[8] and the SPSDMK[4, 9] Hamiltonians. Dominant contributions come from spin-dipole transitions. Calculated results are found to be en- 

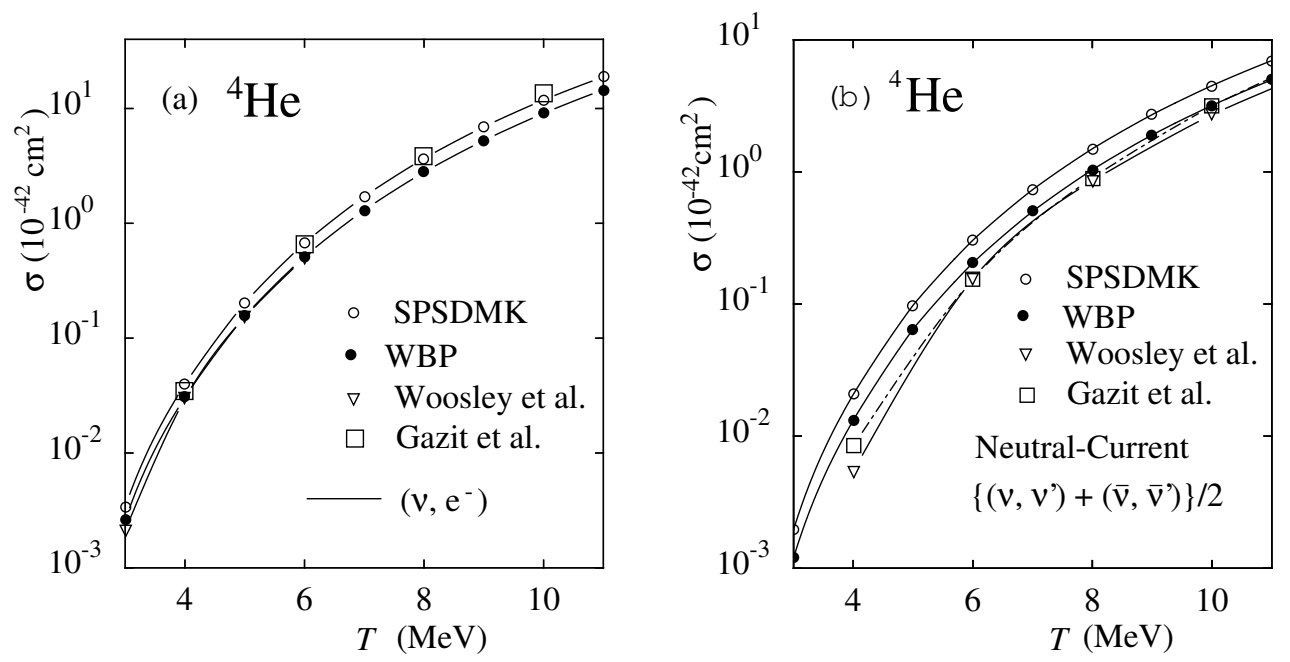

Figure 2: Calculated charge-exchange (a) and neutral current (b) reaction cross sections for ${ }^{4} \mathrm{He}$ induced by supernova neutrinos with temperature $T$ obtained by shell model calculations with the use of the WBP and SPSDMK Hamiltonians. Previous results in ref. [7] and recent ones in ref. [10] are also shown for comparison.

hanced compared with the previous calculations[7]. They are also enhanced compared with the recent ab-initio calculations with AV8' interaction[10] for the neutral current reactions. In case of the charge-exchange reactions, cross sections by ref. [10] are larger than those by the shell model calculations except for the SPSDMK Hamiltonian at $T \leq 6 \mathrm{MeV}$. The cross sections in ref. [10] have steeper temperature dependence than those by the shell model cases. When we take into account the effects of the spreading in the spin-dipole strength, the temperature dependence of the cross sections gets closer to those in ref. [10]. In case of ${ }^{4} \mathrm{He}$, proton and neutron knock-out processes are the dominant ones for both the charge-exchange and the neutral current reactions.

In the nucleosynthesis path of light elements ${ }^{7} \mathrm{Li}$ and ${ }^{11} \mathrm{~B}$ during supernova explosions, the reactions ${ }^{4} \mathrm{He}\left(v, v^{\prime} \mathrm{p}\right){ }^{3} \mathrm{H}$ is important for the production of ${ }^{7} \mathrm{Li}$ as well as ${ }^{11} \mathrm{~B}$ through ${ }^{7} \mathrm{Li}(\alpha, \gamma)$ ${ }^{11} \mathrm{~B}$ in the He/C layer. The reaction ${ }^{12} \mathrm{C}\left(v, v^{\prime} \mathrm{p}\right){ }^{11} \mathrm{~B}$ is important for the production of ${ }^{11} \mathrm{~B}$ in the $\mathrm{O} / \mathrm{Ne}$ and $\mathrm{O} / \mathrm{C}$ layers. The enhancement of the $v-{ }^{4} \mathrm{He}$ and $v-{ }^{12} \mathrm{C}$ reaction cross sections is found to lead to the increase of the production yields of ${ }^{7} \mathrm{Li}$ and ${ }^{11} \mathrm{~B}$ during supernova explosions [5] about by $11 \%$ and $11 \%$ ( $75 \%$ and $38 \%$ ), respectively, in case of WBP+SFO (SPSDMK+PSDMK2) compared with those for the previous case[11].

When there are neutrino oscillations, charge-exchange reactions on ${ }^{4} \mathrm{He}$ and ${ }^{12} \mathrm{C}$ become important and ${ }^{7} \mathrm{Li}$ and ${ }^{11} \mathrm{~B}$ are produced through the production of ${ }^{7} \mathrm{Be}$ and ${ }^{11} \mathrm{C}$. The yield ratio $N\left({ }^{7} \mathrm{Li}\right) / N\left({ }^{11} \mathrm{~B}\right)$ becomes sensitive to the mixing angle $\theta_{13}$ for the normal neutrino mass hierarchy, which can be used to determine the lower limit of $\theta_{13}$ as well as if the neutrino mass hierarchy is normal or inverted[11, 12].

Besides ${ }^{7} \mathrm{Li}$ and ${ }^{11} \mathrm{~B}$, light elements such as ${ }^{10} \mathrm{Be},{ }^{10} \mathrm{~B},{ }^{9} \mathrm{Be}$ and ${ }^{6} \mathrm{Li}$ are also produced by neutrino processes. ${ }^{10} \mathrm{Be}$ is mainly produced through ${ }^{12} \mathrm{C}\left(v, v^{\prime} x\right){ }^{10} \mathrm{Be}$ in both the O-rich and $\mathrm{He} / \mathrm{C}$ layers. Charge-exchange reactions ${ }^{12} \mathrm{C}\left(\bar{v}_{e}, e^{+} x\right){ }^{10} \mathrm{Be}$ are also important if the temperature of $\bar{v}_{e}$ is large. ${ }^{10} \mathrm{~B}$ is produced mainly by ${ }^{12} \mathrm{C}\left(v, v^{\prime} x\right){ }^{10} \mathrm{~B}$ in the O-rich layer. Most of ${ }^{9} \mathrm{Be}$ is produced 

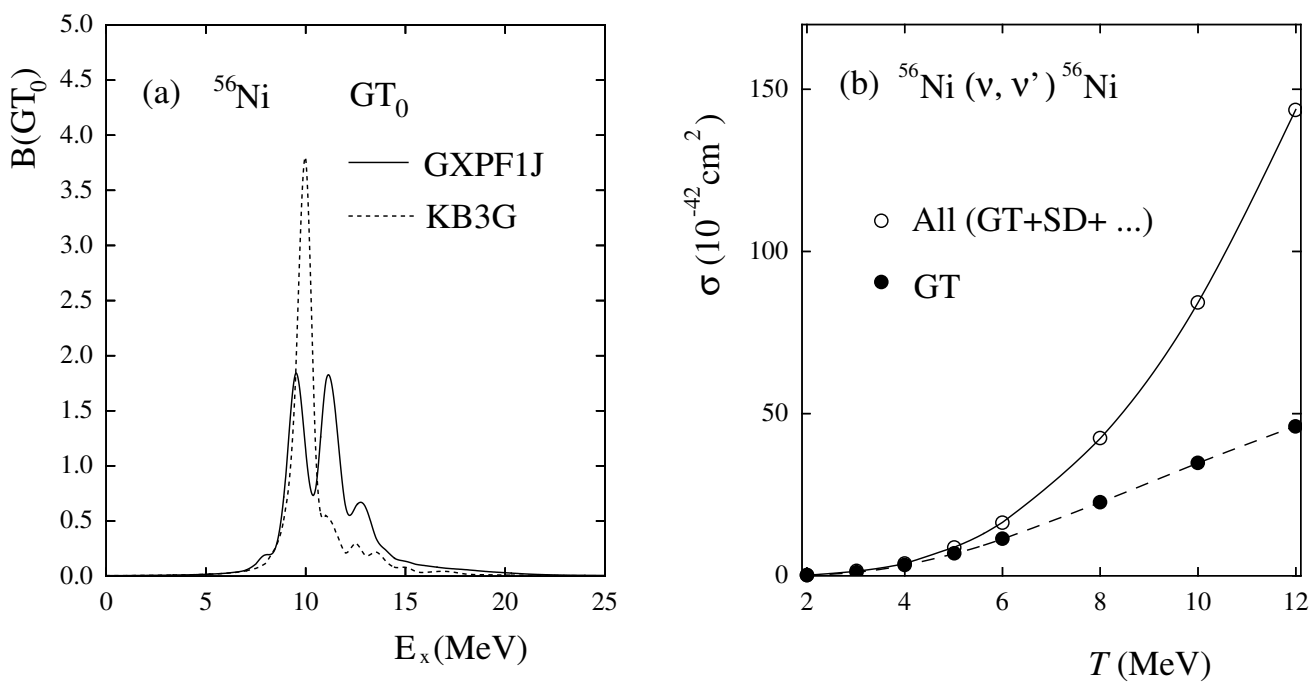

Figure 3: (a) Calculated non-charge-exchange $\mathrm{B}\left(\mathrm{GT}_{0}\right)$ values for ${ }^{56} \mathrm{Ni}$ obtained by GXPF1J and KB3G. (b) Neutral current reaction cross sections for ${ }^{56} \mathrm{Ni}$ induced by supernova neutrinos with temperature $T$. The GT contribution is obtained by GXPF1J.

through ${ }^{12} \mathrm{C}\left(v, v^{\prime} x\right){ }^{9} \mathrm{Be}[12] .{ }^{6} \mathrm{Li}$ is produced through ${ }^{4} \mathrm{He}\left(v, v^{\prime} d\right){ }^{2} \mathrm{H}(\alpha, \gamma){ }^{6} \mathrm{Li},{ }^{12} \mathrm{C}\left(v, v^{\prime} x\right){ }^{6} \mathrm{Li}$, and ${ }^{9} \mathrm{Be}(p, \alpha){ }^{6} \mathrm{Li}$.

\section{Neutrino-Induced Reactions on Ni Isotopes and Production of Mn in Population III Supernovae}

Neutrino-induced reaction on $\mathrm{Fe}$ and $\mathrm{Ni}$ isotopes have been studied with the use of a new shellmodel Hamiltonian for the $f p$-shell, GXPF1J [13]. The Hamiltonian can reproduce GT strength in ${ }^{58} \mathrm{Ni}$ as well as $\mathrm{M} 1$ strengths in ${ }^{48} \mathrm{Ca},{ }^{50} \mathrm{Ti},{ }^{52} \mathrm{Cr}$ and ${ }^{54} \mathrm{Fe}[14,13]$.

Neutral current reactions on ${ }^{56} \mathrm{Ni}$ are studied. The fragmentation of the $\mathrm{GT}_{0}\left(\mathrm{~T}_{f} \geq \mathrm{T}\right)$ strength is generally more pronounced than the charged-current case for the GXPF1J. Calculated GT strength is shown in Fig.3(a) and compared with that for KB3G [15]. Neutral current reaction cross sections induced by supernova neutrinos are shown in Fig. 3(b) for both the GT and the total transitions. Contributions other than the GT transitions are obtained by RPA with the same universal quenching factor of $g_{A}^{e f f} / g_{A}=0.74$. The GT transition is dominant at low neutrino temperature $(T<6 \mathrm{MeV})$, while the contributions from the spin-dipole transitions get larger for higher temperature, $T>9$ $\mathrm{MeV}$.

Now, we study particle knock-out processes in neutral current reactions on ${ }^{56} \mathrm{Ni}$, and discuss production of ${ }^{55} \mathrm{Mn}$ through a path, ${ }^{56} \mathrm{Ni}\left(v, v^{\prime} p\right){ }^{55} \mathrm{Co}\left(e^{-}, v_{e}\right){ }^{55} \mathrm{Fe}\left(e^{-}, v_{e}\right){ }^{55} \mathrm{Mn}$ in supernova explosions. Reaction cross sections are obtained for both GXPF1J and KB3G Hamiltonians and compared with previous nucleon knock-out cross sections[16]. Proton knock-out cross sections are found to be much enhanced for the GXPF1J case. This is due to the fact that the strength is more fragmented and a large fraction of the strength is found in the proton emission channel $\left(\mathrm{E}_{x} \geq 10\right.$ $\mathrm{MeV}$ ) in case of the GXPF1J Hamiltonian (see Fig. 3(a)). Note that the transition to the ground state of ${ }^{55} \mathrm{Co}\left(7 / 2^{-}\right)$at $\mathrm{E}_{x}=7.2 \mathrm{MeV}$ is hindered due to its $f$-wave nature. 
Production yields of elements during supernova explosions for a population III star with mass $15 M_{\odot}[17]$ are investigated. Total $v$ energy of $E_{v}=3 \times 10^{53}$ ergs and $v$ temperatures of $\left(T_{v_{e}}, T_{\bar{v}_{e}}\right.$, $\left.T_{v_{\mu, \tau}}\right)=(4,4,6) \mathrm{MeV}$ are assumed. Cross sections of ref. [16] are used except for neutral current reactions on ${ }^{56} \mathrm{Ni}$. Enhancement of the production yield of ${ }^{55} \mathrm{Mn}$ is obtained when the neutrino processes are included as shown in Table 1. The yield for the GXPF1J Hamiltonian is enhanced compared with those for other Hamiltonians. Enhancement of the production yield of ${ }^{59} \mathrm{Co}$ is also obtained.

\begin{tabular}{lcccccc}
\hline Model & GXPF1J & GXPF1J $\times 2$ & KB3G & KB3G $\times 2$ & HW02 & No $v$ \\
\hline$M\left({ }^{55} \mathrm{Co}\right) /\left(10^{-4} M_{\odot}\right)$ & 4.16 & 5.00 & 3.56 & 4.00 & 3.80 & 2.29 \\
$M(\mathrm{Mn}) /\left(10^{-4} M_{\odot}\right)$ & 4.31 & 5.16 & 3.72 & 4.16 & 3.96 & 2.30 \\
{$[\mathrm{Mn} / \mathrm{Fe}]$} & -0.25 & -0.17 & -0.32 & -0.27 & -0.29 & -0.53 \\
\hline
\end{tabular}

Table 1: Production yields of Mn as well as the logarithmic value of the yield ratio over Fe relative to the solar abundances [ $\mathrm{Mn} / \mathrm{Fe}$ ] in a supernova explosion model of a population III star with $15 \mathrm{M}_{\odot}$

\section{References}

[1] T. Otsuka, R. Fujimoto, Y. Utsuno, B. A. Brown, M. Honma and T. Mizusaki, Phys. Rev. Lett 87, 082502 (2001).

[2] T. Suzuki, R. Fujimoto and T. Otsuka, Phys. Rev. C 67, 044302 (2003).

[3] S. Cohen and D. Kurath, Nucl. Phys. 73, 1 (1965).

[4] D.J. Millener and D. Kurath, Nucl. Phys. A255, 315 (1975).

[5] T. Suzuki, S. Chiba, T. Yoshida, T. Kajino and T. Otsuka, Phys. Rev. C 74, 034307 (2006).

[6] T. E. Drake, E. L. Tomusiak and H. S. Caplan, Nucl. Phys. A118, 138 (1968);

A. Yamaguchi, T. Terasawa, K. Nakahara and Y. Torizuka, Phys. Rec. C 3, 1750 (1971);

C. Gaarde et al., Nucl. Phys. A422, 189 (1984).

[7] S. E. Woosley, D. H. Hartmann, R. D. Foffman and W. C. Haxton, Astrophys. J. 356, 272 (1990).

[8] S. E. Warburton and B. A. Brown, Phys. Rev. C 46, 923 (1992).

[9] OXBASH, B. A. Brown, A. Etchegoyen and W. D. M. Rae, MSU Cyclotron Laboratory Report No. 524 (1986).

[10] D. Gazit and N. Barnea, Phys. Rev. C 70, 048801 (2004); Phys. Rev. Lett 98, 192501 (2007).

[11] T. Yoshida et al., Phys. Rev. Lett 96, 091101 (2006); Astrophys. J. 649, 319 (2006).

[12] T. Yoshida, T. Suzuki, S. Chiba, T. Kajino, H. Yokomakura, K. Kimura, A. Takamura and D. H. Hartmann, Astrophys. J., in press (2008).

[13] M. Honma et al., Phys. Rev. C 65, 061301 (2002); ibid. C 69, 034335 (2004); Journal of Physics: Conference Series 20, 7 (2005).

[14] Y. Fujita et al., Eur. Phys. J A 13, 411 (2002).

[15] A. Poves, J. Sánchez-Solano, E. Caurier and F. Nowacki, Nucl. Phys. A694, 157 (2001).

[16] R. D. Hoffman and S. E. Woosley, Neutrino interaction cross sections and branching ratios, 1992, http://www-phys.llnl.gov/Research/RRSN/nu-csbr/neu-rate.html

[17] T. Yoshida, H. Umeda and K. Nomoto, Astrophys. J 672, 1043 (2008). 